\author{
Voix plurielles \\ Volume 5, Numéro 1 : mai 2008
}

Ilaria Vitali

\title{
La bibliothèque de Shéhérazade en voyage. Reprises et réécritures d'un livre-édifice dans la littérature algérienne contemporaine
}

Citation MLA : Vitali, Ilaria. «La bibliothèque de Shéhérazade en voyage. Reprises et réécritures d'un livre-édifice dans la littérature algérienne contemporaine.»Voix plurielles 5.1 (mai 2008). 


\title{
La bibliothèque de Shéhérazade en voyage. Reprises et réécritures d'un livre-édifice dans la littérature algérienne contemporaine
}

\author{
Ilaria Vitali \\ Université de Bologne - Université Sorbonne-Paris IV
}

Mai 2008

\section{Résumé}

Au carrefour de l'Orient et de l'Occident, la littérature algérienne de langue française a su s'approprier la bibliothèque du recueil des Mille et une Nuits pour en faire un espace scripturaire complexe. Véritable livre-édifice riche en symboles, le célèbre recueil a construit par sa structure un ensemble foisonnant de textes qui ont enchanté le lecteur et ont fait l'objet d'innombrables remaniements et emprunts. Il suffit de penser aux romans d'Assia Djebar, de Leïla Sebbar et plus récemment à ceux de Salim Bachi et de Mourad Djebel.

Nous proposons d'explorer l'étoilement de cette bibliothèque dans la littérature algérienne contemporaine, qui a su exploiter les étagères des Mille et une Nuits comme un véritable espace à habiter et à réinventer.

\section{Au commencement, une bibliothèque}

Véritable édifice riche en symboles, le recueil des Mille et une Nuits construit par sa structure un ensemble foisonnant de textes qui enchantent le lecteur. Mais d'où viennent ces histoires à l'imagination flamboyante?

Contrairement à ce que l'on pourrait penser, la bibliothèque « virtuelle » de Shéhérazade est à l'origine une bibliothèque bien réelle. Car la «Sultane des aubes » n'invente pas, elle lègue les histoires qu'elle connaît: «Elle avait dévoré bien des livres : annales, vies de rois anciens, histoire des peuples passés, ouvrages de médecine. On dit qu'elle avait réuni mille livres touchant à ces peuples, aux rois de l'Antiquité et à leurs poètes. » (Les Mille et Une Nuits, t. I, 41.)

Shéhérazade est donc porteuse des paroles de sa bibliothèque, car « le statut qu'elle revendique est celui de transmetteuse, et justement chacune de ses histoires commence par un «protocole d'ouverture », un syntagme rituel qui la rattache à une voix anonyme et mystérieuse : «On raconte » (yuhkâ), ou bien : «Il m’est parvenu » (balaghanî)». (Kilito 14) 
L'origine de ces contes, précise Kilito, n'est pourtant jamais prise en compte : ils sont tout simplement là, connus et racontés de tout temps. Ce qui est cependant souligné avec force est le fait qu'ils n'ont pas été transmis dans l'oralité, mais qu'ils sont le fruit d'une œuvre de lecture.

Shahrazâd n'a cependant pas recueilli ses contes à la faveur d'une transmission orale, mais dans les livres. Ses seuls maîtres sont des livres, au nombre de mille, où elle a appris la médecine, la poésie, l'histoire et les dits des sages et des rois. C'est dans cette immense mémoire écrite qu'elle a puisé la matière de ses récits, de l'enseignement qu'elle a dispensé à Shâhriyâr, nuit après nuit. Au commencement, une bibliothèque. (Kilito, 14-5).

Il y a deux termes principaux à retenir au préalable dans ce passage : «livres » et «mémoire ». L'importance des livres et de leur transmission dans les contes des Nuits, ainsi que dans la constellation des textes qui s'y inspirent, est manifeste.

La «bibliothèque » de Shéhérazade est donc un récipient, un réservoir, mais aussi un réceptacle, un miroir convexe qui reflète en abyme sa propre histoire, jusqu'au point de pousser Borges à imaginer la nuit centrale du recueil, dans laquelle Shéhérazade, par une distraction magique du copiste, raconte sa propre histoire «qui embrasse toutes les autres, qui monstrueusement - s'embrasse elle-même ». (Borges 68). ${ }^{1}$ L'idée de l'infinité, évoquée par le titre même de l'ouvrage, qui rappelle par la finitude d'un numéro bien précis - 1001 - l'illimité, à mené la bibliothèque de Shéhérazade à voyager dans le temps et l'espace.

Par ailleurs, cet infini dédaléen évoque également l'image du labyrinthe. Dans son essai Il labirinto tra Medioevo e Rinascimento, Umberto Eco concentrait sa réflexion sur trois des métaphores les plus anciennes, puissantes et omniprésentes dans la littérature, qui remettent sans cesse en cause l'identité de l'homme et sa représentation de la réalité. La première envisageait le monde comme un livre, la deuxième le livre comme un monde, et la troisième le monde - et le livre - comme un labyrinthe.

L'infini labyrinthique des Mille et une Nuits, prévu en quelque sorte par la structure du recueil lui-même, a porté cette somme à sa dissémination dans les siècles. Ne pouvant pas analyser toute la constellation de textes qui se sont inspirés du recueil, nous proposons d'étudier ses reprises plus récentes dans quelques romans-clefs de la littérature algérienne de langue française. Au carrefour de l'Orient et de l'Occident, cette littérature a su s'approprier la bibliothèque du célèbre recueil pour en faire un édifice scripturaire complexe, qui dit, entre autres, l'opposition entre l'espace traditionnel et l'espace colonisé, guidée par les dynamiques antagonistes d'ouverture et de clôture, d'intérieur et d'extérieur.

À la lumière de ces dynamiques sous-jacentes il est intéressant d'explorer l'étoilement de la bibliothèque de Shéhérazade dans la littérature algérienne contemporaine, qui a exploité les étagères et les couloirs labyrinthiques des Nuits comme un véritable espace à habiter et à réinventer.

Nous pensons notamment aux romans d'Assia Djebar, de Leïla Sebbar et plus récemment à ceux de Salim Bachi et de Mourad Djebel, qui constituent le corpus que nous analyserons au cours de cet article. 


\section{Naissance et étoilement de la bibliothèque des Milles et une Nuits}

De nombreuses études ont été consacrées à déterminer l'origine des Nuits, qui demeure néanmoins chargée de mystère : un écrit arabe ancien, le Kitab al-Fihrist, rédigé en 987, relate l'existence d'un volume persan contenant l'histoire de Shahrâzâd, intitulé Hezar Efsane (Les Milles Contes) dont pourtant il n'existe nulle trace. De fait, les manuscrits arabes n'ont été retrouvés que partiellement et ont eux-mêmes des sources différentes. Par l'emploi du préfixe «Shah», «Roi » en persan, les noms de Shahrâzâd (Shéhérazade en français) et de Shâhriyâr révéleraient l'origine persane des contes. Cependant, d'autres éléments, tels que le fait de retarder la mort en contant des fables, les métamorphoses en animaux, et les génies demi-dieux font référence au polythéisme hindou et témoigneraient d'une origine indienne, remontant au III ${ }^{\mathrm{e}}$ siècle. L'hypothèse veut donc que les contes soient nés en Inde et que, par voie orale, ils auraient atteint la Perse où un premier recueil, le Hezar Efsane aurait été écrit. Ce recueil se serait ensuite propagé dans le monde arabe grâce, entre autres, aux marchands avides de récits pour briser la monotonie de leurs voyages. Autour du VIII ${ }^{\mathrm{e}}$ siècle, les conteurs arabes auraient traduit le livre et répandu ces histoires en les «arabisant», et en y ajoutant un bon nombre de contes, tout en conservant plusieurs éléments du texte primitif. Parmi les éléments arabes présents dans le recueil, mentionnons les éloges au Prophète, la forte présence des références toponymiques arabes (Bagdad, Le Caire, Bassora, Damas), et les renvois à des poètes célèbres, à des califes et à des savants du monde arabe.

Ainsi s'est constituée la bibliothèque des Nuits.

Par un jeu continu de reprises, d'emprunts, de résurgences et de réinterprétations, ce livreédifice figure comme le lieu privilégié d'une rencontre entre l'Orient arabo-islamique et l'Occident, allant plus loin qu'un jeu stérile d'auto-contemplation, en mettant à jour un processus de réécriture ininterrompu. Au sein de ce mouvement, le complexe entrelacs de relations que l'imaginaire littéraire du $\mathrm{XX}^{\mathrm{e}}$ siècle entretient avec le recueil des Nuits, peut aller «d'une fidélité-calque à une originalité impulsant une œuvre nouvelle. » (Chaulet-Achour 8). Cependant, la figure de la conteuse, qui retarde sa mort par la narration des contes, demeure sans doute l'élément le plus retravaillé.

\section{L'exploitation de la bibliothèque de Shéhérazade pour raconter les femmes du Maghreb}

C'est précisément par la figure de Shéhérazade que des romancières comme Assia Djebar et Leïla Sebbar introduisent en Occident toute une tradition orientale en l'apprivoisant, en l'asservissant à leurs voix nouvelles. Figure multiple, Shéhérazade se prête sans cesse au remaniement et offre aux romancières la matière pour rebrousser chemin, revenir en arrière, autant sur les enjeux que sur les pièges d'un Orient trop souvent mal connu et mal compris. Shéhérazade devient ainsi la voix dans un univers muet, celle qui sauva ses congénères, héroïne triomphante, dans un monde qui s'organise en principe autour de la figure de "l'homme », de «l'ennemi $»^{2}$. Elle incarne la capacité de raconter, de nommer, de dire et de se dire.

«Écrivain-femme porte parole des femmes séquestrées (Chikhi, 2007 14), Assia Djebar a exploité ce mythe protéiforme pour libérer les femmes algériennes, enfermées «dans leur 
appartement ». Dialoguant avec la tradition culturelle occidentale et orientale, ses romans se distinguent par la primauté exceptionnelle qu'ils accordent au féminin.

Second volet du quatuor romanesque à vocation autobiographique commencé avec $L$ 'amour, la fantasia, le roman Ombre sultane semble être un appel à l'intention de ces femmes en attente, «prisonnières résignées d'un lieu clos », invitation à ôter leur voile physique et symbolique. ${ }^{3}$

Évoquant le fait que la «sultane des aubes » n'aurait pas survécu sans l'aide de sa sœur Dunyazad, la narratrice de Djebar, Isma, Shéhérazade contemporaine, porte secours à Hajila, sa coépouse, en l'aidant ainsi à sortir de l'isolement.

La voix comme libération est ensuite reprise par Djebar dans un autre ouvrage, Loin de Médine, roman écrit dans l'urgence et qui vient interrompre la rédaction du quatuor, dans lequel l'auteure examine la situation des femmes depuis les origines de l'Islam, dans le but de rétablir leur rôle de premier plan aussi bien dans la religion que dans l'Histoire, en en faisant l'aboutissement de tout principe de vie. Pour rétablir la mémoire ancienne, Djebar tisse une nouvelle histoire, en exploitant la figure des femmes rawiyates ${ }^{4}$, «les transmetteuses », et dévoile en creux que la parole est un pouvoir et que la «voix » peut devenir «voie » :

Du coup, la fiction contamine l'Histoire ou plutôt la corrige car ces femmes ressuscitées, ces femmes qui ont connu directement ou indirectement le Prophète s'emparent de la «parole vive » et devenues paradoxalement personnages romanesques puis narratrices, profèrent la vérité historique, vérité que le discours officiel ou qu'Assia Djebar identifie comme la Tradition a ensevelie. (Regaieg 2000)

Reconsidérant la place de la femme dans la société musulmane, Djebar, narratrice-historienne, opère en même temps une renégociation des places dans le processus de communication littéraire, en invitant le lecteur à y jouer un rôle actif, à se frayer un chemin dans un récit incapable de séparer le fictionnel de l'historique, dans une écriture entre deux rivages qui ne peuvent être opposés.

Dessiné en filigrane tout le long de son itinéraire littéraire, le mythe de Shéhérazade permet à Djebar de retracer le parcours des femmes qui va de la claustration à la liberté, un chemin qui se termine dans le recueil de nouvelles Femmes d'Alger dans leur appartement, qui semble finalement «libérer » les femmes de leur isolement. ${ }^{5}$ D'après Winifred Woodhull, malgré cette libération, l'œuvre de Djebar demeure pourtant «pessimiste », contrairement, par exemple, à celle de Fatima Mernissi :

Yet even if it offers itself as an instrument of Algerian women's liberation, Djebar's text reinscribes a pessimistic view of her maghrebians sisters that many feminists, such as Fatima Mernissi, contest, emphasizing not their mutilation and dispossession but rather their capacity to speak and act on their on behalf today. (Woodhull 84.) 
Djebar n'est pourtant pas la seule à vouloir faire sortir les femmes d'Alger de leur appartement en exploitant la bibliothèque de Shéhérazade. Dans Fatima ou les Algériennes au square (1981), ou encore dans Parle mon fils, parle à ta mère (1985), Leïla Sebbar attribue une voix aux femmes immigrées de la première génération, en reconstruisant leur mémoire - ainsi que la mémoire collective de tout un peuple - par une sorte de bricolage identitaire.

C'est pourtant dans sa trilogie de "Shéhérazade ${ }^{6}$ que Leïla Sebbar explore les richesses et les rouages d'un Orient qui fascine et repousse à la fois, dont la représentante est la jeune beurette Shérazade, qui insiste sur la prononciation non-orientale de son prénom, et arrive jusqu'à adopter des pseudonymes. ${ }^{7}$ Nomade et picaresque, la fugitive Shérazade questionne les mythes du passé pour construire son identité : en traçant une topographie de la tradition elle finit par découvrir une cartographie nouvelle.

Si le premier roman de la trilogie pose les cadres de la série, le deuxième, Les carnets de Shéhérazade, continue cet itinéraire fictionnel qui veut reconstruire les liens et les contrastes entre deux cultures en présence. Cette reconstruction au carrefour de deux mondes, comporte une réflexion qui se fonde d'une part sur la reprise d'un orient légendaire, et de l'autre sur celle d'une France également mythique. Dans ce processus créatif, l'imagination occupe une place de premier plan. A ce sujet, Inge Boer écrit :

Sherazade and her friends suffer not from national trauma per se, but from their inability to memorialize and mourn Algeria's traumatic past. Denied acces to the past, Sherazade is both limited to, and freed by, her imagination. She must invent the community of Algeria and its implications for her identity. (Boer 33).

Ce besoin de renouer les fils de l'histoire, se lie, chez Sebbar, à la volonté de fonder une nouvelle identité féminine, entre tradition et modernité, entre Orient et Occident, dans l'isthme de l'entre-deux. Dans cette quête des origines le passé demeure pourtant lointain et les qualités de la conteuse mythique sont impossibles à atteindre. A ce sujet, il est intéressant de remarquer que dans le dernier volet de la trilogie, Le fou de Shérazade, la seule fois où la protagoniste prononce son nom comme celui de l'authentique conteuse des Nuits - «Shé-hér-azade », à la façon orientale, avec l'insertion de la syllabe médiane «her »- elle est soudainement incapable de raconter, et son auditoire de l'écouter :

- C'est quoi ton nom?

- Shéhérazade.

- Quoi ? disent ensemble les hommes.

- Shéhérazade.

Elle dit son nom comme on prononce celui de la sultane des Mille et une Nuits.

- Vous entendez ? les hommes parlent tous à la fois.

- On ne se trompait pas, une maison pour une sultane, qui va vous raconter des histoires la nuit, dans la langue la plus belle, la plus pure...Ah ! Quelles nuits... Quelles nuits ! (Sebbar 1991, 19) 
Et, pourtant, ils seront déçus, car ils ne seront pas à même de comprendre son discours « hybride », sa langue arabe parsemée de mots français : «Qu'est-ce qu'elle raconte encore ? On comprend à peine ce qu'elle dit. Quelle langue bâtarde elle parle ? Faites-la taire. » (p. 37) ${ }^{8}$

Noyau fécond, la scénographie de la bibliothèque des Nuits ne s'épuise pas, "ouverture d'une scène double, elle inspire l'infinité sémantique, romanesque et poétique. . . (Chikhi 2007 79)

Et pourtant, dans cette constellation littéraire de reprises, dans cet agrégat de textes multiples, «les nuits se suivent mais ne se ressemblent pas ; nostalgiques, quêteuses, ou critiques chez Boudjedra, elles se font soupçonneuses chez Khatibi, et deviennent motif d'écriture. » (p. 116) Car les hommes-écrivains aussi se sont intéressés à la conteuse et à son livre-édifice. Après tant de femmes qui ont consacré leur œuvre à se mettre dans la peau de Shéhérazade - ou à en sortir , quelques hommes ont accepté de prendre la relève. Leur regard sur la sultane des Nuits est différent, leurs reprises de la bibliothèque le sont aussi.

\section{La bibliothèque de Shéhérazade vue par les hommes}

De toute évidence, Shéhérazade demeure assimilée à l'extraordinaire capacité de raconter. Voici ce qu'écrit Tahar Ben Jelloun :

J'adore raconter des histoires. C'est un métier et une passion. . . Le principe littéraire le plus fondamental de tous les temps, c'est celui des Mille et une nuits. Raconte-moi une histoire ou je te tue... Nous sommes condamnés à raconter des histoires sous peine de disparition. Et une société sans romanciers, sans créateurs, sans raconteurs d'histoires, est une société déjà morte. (Ben Jelloun 107)

Corps-récit immémorial, Shéhérazade est conçue par Khatibi au niveau purement théorique : «nul besoin de l'imaginer comme corps réel, nul besoin de reconstruire cet Orient de pacotille » (Khatibi 11). De prime abord, Khatibi opère une séparation inhérente au corps-conducteur de Shéhérazade :

D’une part, le corps « réel, tatoué de perles et de parfums enivrant dans un harem de paradis où dansent et partouzent les princes (les principes), les concubines et les esclaves en un coït sériel... ». Ce corps, appartenant à un «Orient de pacotille», est à écarter, à oublier, à refouler. D'autre part, le corps «théorique », celui qu'elle-même a mis en jeu sur une scène «hautement théorique », parce que «pensée somptueuse d'un principe absolu, et somptueuse dans son luxe narratif - récits de récits - pour envahir, enchanter et ravir, comme si le narrataire était un mort ensorcelé ». (Khatibi 11 ) 
Dans les mots de Khatibi, Shéhérazade devient ainsi la théorie fastueuse du récit, le principe du conte comme séduction absolue, principe de la littérature tout court. Le titre même de l'ouvrage qui la voit protagoniste s'étoffe dans une poche d'ombre magique, dans l'interstice entre le sommeil et la veille, entre le jour et la nuit, la fiction et la réalité, se voulant ouverture vers un infini romanesque qui devient ainsi motif d'écriture, objet d'écriture.

Par ailleurs, l'essence double de Shéhérazade permet une double - voire multiple interprétation de sa figure. Si d'une part elle consolide la prise de conscience des «femmes séquestrées » et se fait porteuse de la fascination imaginative du récit, de l'autre, elle est aussi « corps à oublier », et quelques romancières des générations plus récentes semblent s'être lassées de la figure de la narratrice orientale, désormais devenue, grâce - ou à cause de - son succès universel, la messagère de cet «orient de pacotille », qui ne fait que recycler et renforcer des clichés abusés auprès du lecteur occidental ${ }^{9}$. Zineb Ali-Benali écrit :

Progressivement le gel a gagné le verbe de Shéhérazade. Son personnage, de ne jamais bouger, est devenu une momie dans une galerie de personnages de cire, un cliché qui a fonctionné - qui fonctionne encore - dans une lecture toute faite. Shéhérazade est devenue le stéréotype de l'orientale, belle et apparemment soumise, personnage-clef des fantasmes masculins.(Ali-Benali : 2004, 217)

En considérant exclusivement le côté « divertissement» de la sultane des aubes, ces auteurs semblent pourtant passer à côté de la complexité des messages qu'elle véhicule, et qui ne peuvent se réduire au fantasme masculin. Car Shéhérazade n'est pas seulement la maîtresse parfaite qui restaure le pouvoir royal grâce à ses contes, elle est aussi l'incarnation de la Qayna, poétesse et musicienne qui entre dans l'espace viril, rendant la parole «incontournable, indépassable, puisque le désir de fiction inoculé à l'homme reste à jamais inassouvi. » (Chikhi 128)

Livre-édifice, la bibliothèque garantit à Shéhérazade non seulement sa survie, mais son immortalité.

\section{Le livre-édifice des Nuits dans l'extrême contemporain}

Grâce à son visage double et aux aspects hétéroclites que sa figure comporte, la sultane des aubes n'a pas été condamnée à l'oubli et revient sans cesse transformée dans les nouvelles voix de la littérature maghrébine. À l'instar de Khatibi et d'Assia Djebar, les auteurs qui suivent, notamment Salim Bachi et Mourad Djebel, considèrent Shéhérazade dans sa capacité imaginative suprême, comme principe prodigieux de l'infinité sémantique et romanesque : «Les contes appellent d'autres contes. Shéhérazade ne s'arrête jamais. Le monde n'est jamais figé. La vérité est multiple. Tout est dans la relation. » (Vitali 372)

Si d'une part, la bibliothèque de Shéhérazade est évoquée sur le plan symbolique, de l'autre on retrouve aussi des reprises de la bibliothèque réelle, physique. Chez Salim Bachi, par exemple, plusieurs genres de bibliothèque émaillent le texte narratif. 
Rappelons ici la première apparition d'une bibliothèque dans Le Chien d'Ulysse, le premier roman de l'auteur. Il s'agit de la bibliothèque d'Ali Khan, professeur de lettres des protagonistes Hocine et Mourad, bibliothèque lourde de sens qui semble in fine crouler «sous son poids de mots imprimés » (Salim Bachi : 2001, 75-6.) A la valeur symbolique de la bibliothèque de ce professeur s'allient les connotations de la toile du Christ mort de Hans Holbein le jeune, situé dans la chambre, à proximité des étagères chargées de livres. Mesurant deux mètres de longueur, le tableau représente le Christ de façon inédite, cadavre humain écrasé par la tombe. D'après la figure de rhétorique de l'ekphrasis, qui fait confluer la description picturale dans l'espace littéraire, le tableau est ainsi retracé :

. . . la bibliothèque croulant sous son poids de mots imprimés, la tranche cassée de chaque livre malmené par un usage frénétique, une reproduction du Christ mort de Holbein le Jeune prenaient un relief surnaturel selon qu'ils étaient ou non exposés au soleil. Le Christ décharné, dans sa tombe, ne jouissait plus d'aucune lumière, une éternité de claustration lui semblait promise. Le miracle ne s'accomplirait jamais, la lumière se refusait au sépulcre. (Salim Bachi : 2001, 75-6.)

Décrit par Hocine, narrateur principal dans Le Chien d'Ulysse, le tableau à côté de la bibliothèque acquiert une valeur grandissante et convie néanmoins un renvoi intertextuel majeur à Dostoïevski, très impressionné par cette peinture qu'il avait pu voir à Bâle, et qui marque les pages de L'Idiot, dans lequel les personnages, et notamment le prince Michkine, en font un commentaire à plusieurs reprises, ce qui ne fait qu'amplifier la valeur de la référence intertextuelle. Par ailleurs, d'après Bernard Aresu :

Combat allégorique entre la lumière vitale et la claustrophobie sépulcrale, de facture brutalement naturaliste, le tableau du Christ mort de Hans Holbein le jeune, dont le récit remarque la violence réformatrice et hétérodoxe, s'impose ainsi comme une référence fictionnellement et métaphysiquement spéculaire, comme ajout pictural densifiant le leitmotiv thanatographique du récit $(C U, \mathrm{p}$. 76). L'objectivité que l'on pourrait qualifier de médico-légale du tableau, sa naturalité cadavérique, son message foncièrement antiidéaliste ne sauraient mieux souligner les angoisses politique et métaphysique que différentes voix ne cessent d'évoquer. (Aresu 180.)

A cette bibliothèque physique s'en ajoutent ensuite plusieurs autres au cours des pages, bibliothèques formées par les noms d'auteurs ou les titres d'ouvrages qui affleurent à la surface du texte, à maintes reprises délivrées au lecteur. C'est le cas de la bibliothèque, cette fois virtuelle, de Hamid Kaïm, autre personnage majeur du Chien d'Ulysse, qui "grandit seul, entouré de livres. » (Bachi 2001, 102) : 
L'Iliade, où Achille combattait et mourait après avoir traîné le corps vaillant et pourtant mort d'Hector. Le chevalier à la triste figure s'élançait contre son dernier moulin. Le père Goriot, sur son lit de misère, attendait vainement la venue de ses chères filles. Il devenait une ville et, sur la colline, lançait comme un refrain un «A nous deux » bien creux. (102)

Dans la bibliothèque de Kaïm, c'est donc d'abord L'Iliade qui a façonné la formation du protagoniste, suivie par Cervantès et Balzac, textes majeurs occidentaux, voire universels. D'ailleurs, le rôle épiphanique des livres est souligné par l'importance attribuée à Ulysses de Joyce, dont Kaïm entame la lecture lors de la mort de la sœur d'Ali Khan. Cyrtha, transformée en Ithaque par les multiples échos de l'Odyssée, devient ainsi Dublin, en se chargeant de toutes les valeurs symboliques que ces rapprochements entraînent.

La bibliothèque de Shéhérazade revient aussi sous d'autres plumes algériennes de la nouvelle génération, comme celle de Mourad Djebel, qui, dans Les cinq et une nuits de Sharazède, raconte l'histoire de l'Algérie depuis l'Indépendance jusqu'aux événements des années 1990 par l'intermédiaire d'une femme :

Ma génération est habitée par une révolte incommensurable, affirme Mourad Djebel, né en 1967, Nous sommes nés après l'indépendance et nous avons connu une sorte de dictature. Notre passage de l'adolescence à l'âge adulte a été ponctué par des explosions - celle des étudiants en 1986, celle de 1988, puis celle qu'on nomme «les événements ». Nous vivions alors la peur au ventre. (Le Monde, «Spécial Salon du Livre» 17 Mars 2006.)

De toute évidence, ce roman présente nombre de ressemblances avec La Kahéna, deuxième roman de Salim Bachi. De même que chez Bachi, la reprise de la figure de Shéhérazade chez Djebel comporte le recyclage d'un autre élément topique des Nuits, à savoir les qualités presque «thaumaturgiques » du livre, son potentiel salvateur, sa capacité de dépasser la réalité, en en faisant oublier ses aspects les plus redoutables. Comme Shéhérazade, les personnages de ces romans luttent pour préserver des histoires de l'oubli, pour retarder leur mort, sabre suspendu au dessus de leur tête.

Comme La Kahéna de Bachi, le roman de Djebel met en scène une structure divisée en plusieurs «nuits ». Du point de vue thématique, les romans des deux auteurs traitent de l'Algérie depuis l'Indépendance jusqu'aux années 1990. Pivot du récit, la protagoniste féminine cherchera à sauver un homme plongé dans une profonde dépression au cours des «années noires », à l'aide du verbe et de la poésie. Comme dans les Nuits, plusieurs récits se mêlent dans la construction romanesque orchestrée par Djebel: au premier degré, l'histoire d'amour entre les deux protagonistes, qui permet de retracer l'histoire de l'Algérie, est ensuite compliquée par les récits des nuits qui renouent avec la tradition des conteuses ancestrales.

Sous l'angle stylistique, Djebel ne se contente pas de phagocyter les règles romanesques traditionnelles et préfère tresser les genres, en insérant ainsi des carnets (les carnets de 
Shâhriyâr), des poèmes et des chansons tirés des Nuits (par exemple, la Chanson de Oum Kalthoum, p. 294). L'intérêt et l'attention pour le texte et tout ce qui l'entoure sont soulignés également par la forte présence des notes, des citations, des traductions qui émaillent le roman, ainsi que par les remarques métacritiques (comme les commentaires sur la traduction des Nuits d'Antoine Galland.)

Par l'analyse de quelques romans-clefs, ce court voyage dans la littérature algérienne francophone nous a montré de quelle manière le livre-édifice de Shéhérazade continue d'influencer les auteurs contemporains. Nous avons pu apprécier de quelle façon ces livres ne figurent pas seulement comme récipients de «toutes les histoires, de l'intégralité du raconté et du racontable » (Kilito 15), mais deviennent aussi des éléments romanesques capables de propulser l'histoire. Ainsi, la bibliothèque de la célèbre conteuse a joué un rôle de premier plan dans la constellation de textes qui s'inspirent des Nuits. En voyageant à travers l'espace et le temps, le livre-édifice de la Sultane des Aubes est devenu le lieu idéal de la rencontre et de l'étoilement des histoires et des savoirs.

\section{Bibliographie}

\section{Euvres Littéraires}

Bachi Salim, (2001). Le Chien d'Ulysse, Paris : Gallimard.

---. La Kahéna, (2003). Paris : Gallimard.

Chouaki Aziz, (1998). Les Oranges, Paris : Les Mille et Une Nuits.

Djebar Assia, (1980). Femmes d'Alger dans leur appartement, Paris : Albin Michel.

---. (1987). Ombre sultane, Paris : Jean-Claude Lattès.

---. (1995). Vaste est la prison, Paris : Albin Michel.

Djebel Mourad, (2005). Les cinq et une nuits de Sharazède, Paris : La Différence.

Sebbar Leïla, (1982). Shérazade, 17 ans, brune, frisée, les yeux verts, Paris : Stock.

---. (1991). Le Fou de Shérazade, Paris : Stock.

\section{Ouvrages critiques}

Bernard Aresu, (2004). "Arcanes algériens entés d'ajours helléniques : Le chien d'Ulysse de Salim Bachi », in Charles Bonn (dir.), Echanges et mutations des modèles littéraires entre Europe et Algérie, Paris : L’Harmattan, 177-187.

Bencheikh Jamel Eddine; André Miquel, (1996). Les Mille et Une Nuits I, II et III, (trad.), Paris : Gallimard.

Galland Antoine (trad.), (1965). Les Mille et Une Nuits, Paris : Flammarion.

Bencheikh Jamel Eddine, Les Mille et Une Nuits ou la parole prisonnière, Paris : Gallimard, 1988. 
Ben Jelloun Tahar, (1995). Tahar Ben Jelloun. Deux cultures, une littérature, entretien avec Pierre Maury, Magazine Littéraire, Paris, no 329.

Bishop Michael, (1996). Thirty Voices in the Feminin, Amsterdam/Atlanta : Rodopi.

Boer Inge E. (2003). After Orientalism: Critical Entanglements, Productive looks, Amsterdam/New York : Rodopi.

Borges Jorge Luis, (1986). Enquêtes, Paris : Gallimard.

«Ces nouvelles écritures nées de la douleur de l'Algérie », Le Monde, «Spécial Salon du Livre », 17 Mars 2006.

Chaulet-Achour Christane (dir.), (2004). Les 1001 Nuits et l'imaginaire du XXe siècle, Paris : L'Harmattan..

Chikhi Beïda, (1996). Assia Djebar, extrait de La littérature maghrébine de langue française, Charles Bonn (dir.), Naget Khadda \& Abdallah Mdarhri-Alaoui, Paris, EDICEF-AUPELF, <http://www.ziane-online.com/assia_djebar/textes/assia_djebar2.htm>.

---. (2007). Assia Djebar. Histoires et fantaisies, Paris : PUPS.

Khatibi Abdelkébir, (1988). Ombres japonaises précédé de Nuits blanches, Montpellier: Fata Morgana.

Kilito Abdelfattah, (1994). L'oil et l'aiguille. Essai sur les Mille et Une Nuits, Paris : La découverte.

Laronde Michel, (1993). Autour du roman beur, Paris : L'Harmattan.

Najiba Regaieg, (2000). "L'histoire sans les femmes, l'histoire des femmes, l'histoire par les femmes dans Loin de Médine d'Assia Djebar », Expressions maghrébines, n 20-21.

Vitali Ilaria, (2007). «Si Shéhérazade ne s'arrete jamais ». Entrevue avec Salim Bachi, en annexe à la thèse Entre les Mille et une nuits et Internet, Université de Bologne/Université Paris IV : 366-72.

Woodhull Winifred, (1993). Transfigurations of the Maghreb: Feminism, Decolonization, and Literatures, Minneapolis : University of Minnesota Press.

${ }^{1}$ Il est sans doute intéressant de remarquer que cette nuit, désignée par Borges comme la $602^{\text {ème }}$, n'existe dans aucune des versions du recueil des Mille et une Nuits (voir à ce sujet A. Kilito 15). Séduit par l'invention borgésienne, même Italo Calvino n'hésite pas à s'attacher à cette fascinante possibilité (voir à ce sujet Livelli di realtà, sous la direction de Piattelli Palmarini, Feltrinelli, 1984, p. 441.)

2 «e'dou», «ennemi ». C'est ainsi que les femmes au hammam définissent leurs maris. Voir A. Djebar, (1995). Vaste est la prison, Paris : Albin Michel, p. 14.

3 «Ombre sultane porte à son paroxysme le sentiment de révolte féminine contre toutes les oppressions, qui se traduit par un encouragement manifeste à changer la société par l'affirmation féminine individuelle d'abord, collective ensuite. » ( Chikhi, Assia Djebar, extrait de La littérature maghrébine de langue française, Charles Bonn (dir.), Naget Khadda \& Abdallah Mdarhri-Alaoui, Paris, EDICEF-AUPELF, 1996, <http://www.zianeonline.com/assia_djebar/textes/assia_djebar2.htm>.

${ }^{4}$ En effet, Djebar paraît entendre par ce terme non seulement les « transmetteurs » de la vie du Prophète, mais plutôt les conteurs de toute la tradition transmise.

${ }^{5}$ Ce que Picasso avait déjà fait en peinture, à travers la revisitation de la toile de Delacroix, comme l'explique bien Djebar dans la postface à son recueil, "Regard interdit, son coupé »: "Picasso renverse la malédiction, fait éclater le malheur, inscrit en lignes hardies un bonheur totalement nouveau. Prescience qui devrait, dans notre 
quotidien, nous guider. », Djebar, (2002). Femmes d'Alger dans leur appartement, Paris : Albin Michel, p. 244.

${ }^{6}$ La trilogie qui voit protagoniste Shérazade est constituée par trois romans : Shérazade, 17 ans, brune, frisée, les yeux verts (1982), Les carnets de Shérazade (1985) et Le fou de Shérazade (1991).

7 Au sujet de la substitution nominale fonctionnelle opérée par la Shérazade de Sebbar, voir M. Laronde, (1993). Autour du roman beur, Paris : L'Harmattan, pp. 197-205.

${ }^{8}$ Voir aussi à ce sujet M. Bishop, (1996). Thirty Voices in the Feminin, Amsterdam/Atlanta : Rodopi, p. 244.

${ }^{9}$ Et pourtant nombreuses sont les reprises qui réduisent Shéhérazade à la femme séductrice. Voir aussi la pièce Les Oranges Aziz Chouaki :

Tiens tout s'arrête, qu'est-ce qui se passe ? Le match s'est arrêté, mais qu'est-ce qui se passe ? Tous les mâles du quartier sont immobiles. Ah, ça y est, c'est Schéhérazade, qui vient du bout de la rue, comme elle est belle... jambes luisantes et bronzées, ses seins moulés dans une casaca blanche, et sa gueule d'amour... Tout le monde bave et bande, normal, faut la voir, Schéhérazade, frimousse brune, sourire marine. (Chouaki 38.) 